\title{
Zur Erinnerung an Heinrich Buess
}

\author{
17.5.1911-31.12.1984
}

Am Silvesterabend 1984 ist, für alle unerwartet, Heinrich Buess an einem akuten Herzversagen gestorben; einen schweren Herzinfarkt hatte er 1968 überstanden. Es war ihm vergönnt, in seinem Haus in Basel in der Obhut seiner Frau sein Leben zu beschließen. Mit ihm ist einer der führenden Schweizer Medizinhistoriker dahingegangen. Der Kanton Basel-Stadt hat ihm freilich, trotz verschiedener Vorstöße der Medizinischen Fakultät, nie eine vollamtliche Professur verliehen; die älteste Universität der Schweiz verzichtet auch heute noch auf den «Luxus» eines eigentlichen Lehrstuhls für Geschichte der Medizin. Das Faktum erregt auswärts immer wieder fast ungläubiges Staunen und hat natürlich auch Heinrich Buess lange zu schaffen gemacht.

25 Jahre lang übte er infolgedessen die Tätigkeit eines Medizinhistorikers neben dem Hauptberuf eines Werksarztes aus. Die CIBA AG war das erste schweizerische Unternehmen, das - 1946 - den Posten eines vollamtlichen Fabrikarztes schuf und damit einen bahnbrechenden Schritt im Bereich der Arbeitsmedizin tat. Buess' Verdienste auf diesem Gebiet sind in der Festschrift zu seinem 70. Geburtstag von seinem Kollegen Hans Leonhard Küng gewürdigt worden ${ }^{1}$; namentlich wies $\mathrm{Küng}$ auf die von Buess nachgewiesenen toxischen Krankheitsbilder der «Chrom-Enteropathie» (1950) und einer leichten hämolytischen Pyrazol-Anämie (1964) hin.

Sein großes wissenschaftliches Arbeitsfeld, die Medizinhistorik, betrat Buess schon mit seiner Dissertation über «Die Wandlungen des Psychogenie-Begriffs» (1937, publ. Hölstein/BL 1940); der gelehrte und geistreiche Basler Neurologe Robert Bing war Initiator und Leiter der Arbeit und sprach in seinem Vorwort die bestimmte Erwartung aus, Buess' Monographie werde dazu beitragen, «dem bedauernswerten Antagonismus zwischen der «neurologischen〉 und der «psychiatrischen〉 Betrachtungsweise hysterischer Reaktionen entgegenzuwirken».

In der Folge bildete sich Heinrich Buess unter Alfred Labhardt am Basler Frauenspital zum Spezialarzt für Geburtshilfe und Gynäkologie aus. Die Geschichte dieser Fächer hat ihn immer wieder beschäftigt; man konsultiere etwa seinen großen Beitrag zur Jubiläumsschrift der Basler Frauenklinik (1970), seine Studien zur Geschichte der Spätgestose, der sog. Eklampsie 
(1971/72), oder über den aus dem Kanton Zürich stammenden Leipziger Geburtshelfer Paul Zweifel (Gesnerus 1974).

Auch in der CIBA fand Heinrich Buess Anregung zur Medizingeschichte: Karl Reucker ${ }^{2}$, der Gründer und Leiter der medizin- und kulturgeschichtlich so gehaltvollen CIBA Zeitschrift, zog den jungen Kollegen schon vor dessen Eintritt in die Firma als Autor heran und unterstützte ihn in seinem eher ungewöhnlichen Entschluß, sich an der Universität Basel für das Fach der Medizingeschichte zu habilitieren, was 1946 geschah. 1956 folgte die Ernennung zum Extraordinarius. Buess" Habilitationsschrift über «Die historischen Grundlagen der intravenösen Injektion» (Aarau 1946) behandelt die einschlägigen Versuche im 17. Jahrhundert. Sie ruft dadurch dem Leser in Erinnerung, wie weit zurück die Bemühungen um diese moderne Behandlungstechnik reichen und an welchen erst später lösbaren Problemen eine frühe Verwirklichung der Idee scheitern mußte.

Damit hatte sich Buess als Kenner und Historiker einer Epoche erwiesen, die ihn persönlich ganz besonders ansprach: die «Barockmedizin», wie er die Heilkunde des späten 16. und des 17. Jahrhunderts, in Übereinstimmung mit Paul Diepgen, zu nennen liebte. Diese Epoche hat gerade in der Schweiz ihre sehr bedeutenden Vertreter gehabt, und Buess hat manchen davon historisch bearbeitet, z.B. den Genfer Théophile Bonet, der durch seine Kompilation pathologisch-anatomischer Befunde zum Wegbereiter Morgagnis wurde.

Es versteht sich von selbst, daß die Geschichte des öffentlichen Gesundheitswesens, der Sozial- und Arbeits-, auch der Militärmedizin ebenfalls zu den von Buess bevorzugten Forschungsgebieten gehörte. Den bahnbrechenden Schweizer Sozialmedizinern des 19. Jahrhunderts, dem St. Galler Laurenz Sonderegger und dem Glarner Fridolin Schuler, galt seine besondere Sympathie.

Heinrich Buess war ein ungemein heimatverbundener Medizinhistoriker. Einen Ruf nach Berlin lehnte er ab. Vor allem aber war es sein konstantes Anliegen, Persönlichkeit, Leben und Werk früherer Schweizer Ärzte darzustellen, ihren Beitrag zur Entwicklung der übernationalen Medizin zu umreißen und einzuordnen. Davon zeugt die von Erwin H. Ackerknecht und ihm gemeinsam herausgebrachte «Kurze Geschichte der großen Schweizer Ärzte» (Bern 1975) sowie - neben vielen Einzelstudien - die riesige Reihe kurzer Biographien, die er mit Hilfe seiner treuen Mitarbeiterin MarieLouise Portmann in «Médecine et Hygiène» unter der Rubrik «Médecins suisses célèbres» veröffentlicht hat. Es war ihm immer wichtig, so wie es hier 
geschah, die Geschichte der Medizin einem weiten Kreis von Ärzten, bei anderer Gelegenheit auch Nichtärzten, zu erschließen. Es bleibt zu bedauern, daß er seine immense Kenntnis der Schweizer Ärzte aller Jahrhunderte nicht noch zu einem umfassenden Werk ausgestaltet hat.

Sehr vieles von Buess' Ideen und Plänen ist im einzelnen von seinen Mitarbeitern und Doktoranden ausgearbeitet worden und in Gemeinschaftswerken oder Dissertationen erschienen. Ich nenne je ein Beispiel: «Die pathologisch-anatomischen Arbeiten bedeutender Schweizer Ärzte 1670-1720» von Susi Joos-Renfer, Marie-Louise Portmann und H. Buess, Basel 1961; «Johann Jakob Wepfer (1620-1695) als klinischer Praktiker» von Pietro Eichenberger, Basel 1969. Beide Werke sind in der von Buess begründeten Schriftenreihe des Verlages Schwabe \& Co. herausgekommen, den «Basler Veröffentlichungen zur Geschichte der Medizin und Biologie». Buess' eigene Arbeiten wie die von ihm geleiteten Dissertationen zeichnen sich durch ihre handwerkliche Solidität aus: sorgfältiger Umgang mit den Quellen, systematische Darlegung der gut belegten Ergebnisse. In erster Linie ging es dem Medizinhistoriker Buess immer darum, die Tatsachen zuverlässig darzustellen und beweisbare Zusammenhänge aufzuzeigen. Spekulative Ausdeutungen und spektakuläre rapprochements waren ihm fremd. Das entspricht nicht nur seinem nüchternen Baselbieter Temperament, sondern auch der Skepsis gegenüber aller «Geschichtsphilosophie», an der wohl jeder teilhat, dem Jacob Burckhardts «Weltgeschichtliche Betrachtungen» wichtig sind.

Beeindruckend war und bleibt die Ausdauer, mit der Heinrich Buess seine Kräfte für die Sache einsetzte, der er sich verpflichtet hatte. Das kam auch unserer Gesellschaft und ihrer Zeitschrift zugute. Dem Vorstand der SGGMN gehörte er von 1955 bis 1977 an; 1971-1977 stand er ihr als Präsident vor. Nach außen wirkte Heinrich Buess zurückhaltend; wer ihm näher kam, erfuhr seine Freundlichkeit und Güte. Wir denken dankbar an ihn zurück.

1 Gesnerus 38 (1981), Heft 1/2, S.6. Dort auch die Bibliographie von Buess' Veröffentlichungen und sein Bild.

2 Siehe Thomas E. Geigy, Dr.med. Dr.med. h.c. Karl Reucker 1890-1961. Zürcher Medizingeschichtliche Abhandlungen Nr. 109, Juris, Zürich 1975.

Prof. Dr. med. H. M. Koelbing, Medizinhistorisches Institut, Rämistraße 71, CH-8006 Zürich 